1 Hacettepe Journal of Mathematics and Statistics

h Volume 46 (2) (2017), $193-198$

\title{
Centralizers and the maximum size of the pairwise noncommuting elements in finite groups
}

\author{
Seyyed Majid Jafarian Amiri* and Hojjat Rostami ${ }^{\dagger}$
}

\begin{abstract}
In this article, we determine the structure of all nonabelian groups $G$ such that $G$ has the minimum number of the element centralizers among nonabelian groups of the same order. As an application of this result, we obtain the sharp lower bound for $\omega(G)$ in terms of the order of $G$ where $\omega(G)$ is the maximum size of a set of the pairwise noncommuting elements of $G$.
\end{abstract}

Keywords: finite group, centralizer, $C A$-group.

2000 AMS Classification: 20D60

Received : 02.02.2016 Accepted : 14.07.2016 Doi : 10.15672/HJMS.20164519332

\section{Introduction and main results}

Throughout this paper $G$ will be a finite group and $Z(G)$ will be its center. For a positive integer $n$, let $Z_{n}$ and $D_{2 n}$ be the cyclic group of order $n$ and the dihedral group of order $2 n$, respectively. For a group $G$, we define $\operatorname{Cent}(G)=\left\{C_{G}(x): x \in G\right\}$ where $C_{G}(x)$ is the centralizer of the element $x$ in $G$. It is clear that $G$ is abelian if and only if $|\operatorname{Cent}(G)|=1$. Also it is easy to see that there is no group $G$ with $|\operatorname{Cent}(G)|=2$ or 3 . Starting with Belcastro and Sherman [7], many authors have investigated the influence of $|\operatorname{Cent}(G)|$ on the group $G$ (see [1], [4], [6], [7], [17-21] and [27-29]). In the present paper, we describe the structures of all groups having minimum number of centralizers among all nonabelian groups of the same order, that is:

*Department of Mathematics, Faculty of Sciences, University of Zanjan, P.O.Box 4537138791, Zanjan, Iran

Email : sm-jafarian@znu.ac.ir

${ }^{\dagger}$ Department of Mathematics, Faculty of Sciences, University of Zanjan, P.O.Box 45371-38791, Zanjan, Iran

Email : h.rostami5991@gmail.com 
1.1. Theorem. Let $G$ be a nonabelian group of order $n$. If $|\operatorname{Cent}(G)| \leq|\operatorname{Cent}(H)|$ for all nonabelian groups $H$ of order $n$, then one of the following holds:

(1) $G$ is nilpotent, $|\operatorname{Cent}(G)|=p+2$ and $\frac{G}{Z(G)} \cong Z_{p} \times Z_{p}$ where $p$ is the smallest prime such that $p^{3}$ divides $n$.

(2) $G$ is nonnilpotent, $|\operatorname{Cent}(G)|=p^{m}+2$ and $\frac{G}{Z(G)} \cong\left(Z_{p}\right)^{m} \rtimes Z_{l}$ where $l>0$ and $p^{m}$ is the smallest prime-power divisor of $n$ such that $p^{m}-1$ and $n$ are not relatively prime.

The following corollary are immediate consequence of Theorem 1.1.

1.2. Corollary. Suppose that $n$ is even and $G$ is a nonabelian group of order $n$. If $|\operatorname{Cent}(G)| \leq|\operatorname{Cent}(H)|$ for all nonabelian groups $H$ of order $n$, then $|\operatorname{Cent}(G)|=4$ or $p+2$ where $p$ is the smallest odd prime divisor of $n$ and also $\frac{G}{Z(G)}$ is isomorphic to one of the following groups:

$$
Z_{2} \times Z_{2}, Z_{p} \times Z_{p}, D_{2 p}
$$

1.3. Remark. We notice that both conditions (1) and (2) of Theorem 1.1 may happen for some positive integer $n$. For example there exist two groups $G_{1}$ and $G_{2}$ of order 54 such that $\left|\operatorname{Cent}\left(G_{1}\right)\right|=\left|\operatorname{Cent}\left(G_{2}\right)\right|=5, \frac{G_{1}}{Z\left(G_{1}\right)} \cong Z_{3} \times Z_{3}$ and $\frac{G_{2}}{Z\left(G_{2}\right)} \cong D_{6}$.

There are interesting relations between centralizers and pairwise noncommuting elements in groups (see Proposition 2.5 and Lemma 2.6 of [1]). Let $G$ be a finite nonabelian group and let $X$ be a subset of pairwise noncommuting elements of $G$ such that $|X| \geq|Y|$ for any other set of pairwise noncommuting elements $Y$ in $G$. Then the subset $X$ is said to have the maximum size, and this size is denoted by $\omega(G)$. Also $\omega(G)$ is the maximum clique size in the noncommuting graph of a finite group $G$. The noncommuting graph of a group $G$ is defined as a graph whose $G \backslash Z(G)$ is the set of vertices and two vertices are joined if and only if they do not commute. By a famous result of Neumann [22] answering a question of Erdôs, the finiteness of $\omega(G)$ is equivalent to the finiteness of the factor group $\frac{G}{Z(G)}$ which follows that $|\operatorname{Cent}(G)|$ is finite. Also,if $G$ has a finite number of centralisers, then it is easy to see that $\omega(G)$ is finite. Various attempts have been made to find $\omega(G)$ for some groups $G$. Pyber [24] has proved that there exists a constant $c$ such that $\left|\frac{G}{Z(G)}\right| \leq c^{\omega(G)}$. Chin [13] has obtained upper and lower bounds of $\omega(G)$ for extra-special groups $G$ of odd order. Isaacs has shown that $\omega(G)=2 m+1$ for any extraspecial group $G$ of order $2^{2 m+1}$ (see page 40 of [11]). Brown in [9] and [10] has investigated $\omega\left(S_{n}\right)$ where $S_{n}$ is the symmetric group on $n$ letters. Also Bertram, Ballester-Bolinches and Cossey gave lower bounds for the maximum size of non-commuting sets for certain solvable groups([5]). Recently authors [17, 20] have determined all groups $G$ with $\omega(G)=5$ and obtained $\omega(G)$ for certain groups. Known upper bounds for this invariant were recently used to prove an important result in modular represention theory ( [13]). In this article we determine the structure of nonabelian groups $G$ of order $n$ such that $\omega(G) \leq \omega(H)$ for all nonabelian groups $H$ of order $n$.

1.4. Theorem. Let $G$ be a nonabelian group of order $n$. If $\omega(G) \leq \omega(H)$ for all nonabelian groups $H$ of order $n$, then one of the following holds:

(1) $G$ is nilpotent, $\omega(G)=p+1$ and $\frac{G}{Z(G)} \cong Z_{p} \times Z_{p}$ where $p$ is the smallest prime such that $p^{3}$ divides $n$.

(2) $G$ is nonnilpotent, $\omega(G)=p^{m}+1$ and $\frac{G}{Z(G)} \cong\left(Z_{p}\right)^{m} \rtimes Z_{l}$ where $l>0$ and $p^{m}$ is the smallest prime-power divisor of $n$ such that $p^{m}-1$ and $n$ are not relatively prime.

Throughout this paper we will use usual notation which can be found in [25] and [15]. 


\section{Proofs of the main results}

The following lemmas are useful in the proof of the main theorem.

2.1. Lemma. Let $G, G_{1}, \cdots, G_{n}$ be finite groups. Then

1. If $H \leq G$, then $|\operatorname{Cent}(H)| \leq|\operatorname{Cent}(G)|$;

2. If $G=\prod_{i=1}^{n} G_{i}$, then $|\operatorname{Cent}(G)|=\prod_{i=1}^{n}\left|\operatorname{Cent}\left(G_{i}\right)\right|$.

Proof. The proof is clear.

In Lemma 2.7 of [4], it was shown that if $p$ is a prime, then $|\operatorname{Cent}(G)| \geq p+2$ for all nonabelian $p$-groups $G$ and the equality holds if and only if $\frac{G}{Z(G)} \cong Z_{p} \times Z_{p}$. In the following we generalize this result for all nilpotent groups.

2.2. Lemma. Let $G$ be a nilpotent group and $p$ be a prime divisor of $|G|$ such that a Sylow $p$-subgroup of $G$ is nonabelian. Then $|\operatorname{Cent}(G)| \geq p+2$ with equality if and only if $\frac{G}{Z(G)} \cong Z_{p} \times Z_{p}$.

Proof. Suppose that $P$ is a Sylow $p$-subgroup of $G$. Then we have $|\operatorname{Cent}(G)| \geq|\operatorname{Cent}(P)| \geq$ $p+2$ by Lemma 2.1(1) and Lemma 2.7 of [4], as wanted.

Now, assume that $|\operatorname{Cent}(G)|=p+2$. Since $G$ is nilpotent, each Sylow $q$-subgroup of $G$ is abelian for each prime divisor $q \neq p$ of $|G|$ by Lemma 2.1(2). Consequently $\frac{G}{Z(G)} \cong \frac{P}{Z(P)}$ which is isomorphic to $Z_{p} \times Z_{p}$ by Lemma 2.7 of [4]. The converse holds similarly.

Recall that a minimal nonnilpotent group is a nonnilpotent group whose proper subgroups are all nilpotent. In 1924, O. Schmidt [26] studied such groups. The following result plays an important role in the proof of Theorem 1.1.

2.3. Lemma. Let $G$ be a minimal nonnilpotent group. Then $\frac{G}{Z(G)}$ is Frobenius such that the Frobenius kernel is elementary abelian and the Frobenius complement is of prime order.

Proof. By Theorem 9.1.9 of [25], we have $G=P Q$ where $P$ is a unique Sylow $p$-subgroup of $G$ and $Q$ is a cyclic Sylow $q$-subgroup of $G$ for some distinct primes $p$ and $q$. Also by Exercise 9.1 .11 of [25], the Frattini subgroups of $P$ and $Q$ are contained in $Z(G)$. It follows that $\frac{P Z(G)}{Z(G)}$ is elementary and $\frac{Q Z(G)}{Z(G)}$ is of order $q$. Since all Sylow subgroups of $\frac{G}{Z(G)}$ are abelian, Theorem 10.1 .7 of [25] gives that $\left(\frac{G}{Z(G)}\right)^{\prime} \cap Z\left(\frac{G}{Z(G)}\right)=\overline{1}$. Since $P=[P, Q]$, we have $\frac{P Z(G)}{Z(G)} \leq \frac{G^{\prime} Z(G)}{Z(G)}$ and so $Z\left(\frac{G}{Z(G)}\right)$ is a $q$-group. On the other hand since $G$ is not nilpotent, $Z\left(\frac{G}{Z(G)}\right)=\overline{1}$. Now it is easy to see that $\frac{G}{Z(G)}$ is a Frobenius group.

2.4. Proposition. Let $\frac{G}{Z(G)}=\frac{K}{Z(G)} \rtimes \frac{H}{Z(G)}$ be a Frobenius group such that $\mathrm{H}$ is abelian. If $Z(G)<Z(K)$, then $|\operatorname{Cent}(G)|=|\operatorname{Cent}(K)|+\left|\frac{K}{Z(G)}\right|+1$ and if $Z(G)=Z(K)$, then $|\operatorname{Cent}(G)|=|\operatorname{Cent}(K)|+\left|\frac{K}{Z(G)}\right|$. Also $\omega(G)=\omega(K)+\left|\frac{K}{Z(G)}\right|$.

Proof. See Proposition 3.1 of [18] and its proof.

Recall that a group $G$ is a $C A$-group if the centralizer of every noncentral element of $G$ is abelian. R. Schmidt [26] determined all $C A$-groups (see Theorem A of [14]). Now we are ready to prove the main result.

Proof of Theorem 1.1.

Suppose that $G$ is a nilpotent group. Since $G$ is not abelian, a Sylow $q$-subgroup of $G$ is not abelian for some prime $q$. It follows from Lemma 2.2 that $|\operatorname{Cent}(G)| \geq q+2$. 
But there exists a nonabelian group $H:=Q \times Z_{\frac{n}{q^{3}}}$ of order $n$ where $Q$ is a nonabelian group of order $q^{3}$ and we see that $|\operatorname{Cent}(H)|=q+2$. Since $G$ has the minimum number of the element centralizer, we must have $|\operatorname{Cent}(G)|=p+2$ and $p$ must be the smallest prime such that $p^{3}$ divides $n$. Also $\frac{G}{Z(G)} \cong Z_{p} \times Z_{p}$ by Lemma 2.2, as wanted.

Now, assume that $G$ is a nonnilpotent group of order $n$. Then there exist two prime divisors $q$ and $r$ of $n$ such that $q$ divides $r^{k}-1$ for some positive integer $k$ by Corollary 1 of [23]. We claim that if $p^{m}$ is the smallest prime-power divisor of $n$ such that $\operatorname{gcd}\left(p^{m}-\right.$ $1, n) \neq 1$, then $|\operatorname{Cent}(G)| \geq p^{m}+2$.

Since $G$ is finite and nonnilpotent, $G$ contains a minimal nonnilpotent subgroup $M$. It follows from Lemma 2.3 that $\frac{M}{Z(M)}$ is Frobenius with the kernel $\frac{K}{Z(M)}$ and the complement $\frac{H}{Z(M)}$. Note that $\left|\frac{K}{Z(M)}\right|=p_{1}^{t}$ and $\left|\frac{H}{Z(M)}\right|=p_{2}$ for some primes $p_{1}$ and $p_{2}$ such that $p_{2} \mid p_{1}^{t}-1$. It follows from Proposition 2.4 that $|\operatorname{Cent}(M)| \geq\left|\frac{K}{Z(M)}\right|+2=p_{1}^{t}+2$. Since $M$ is a subgroup of $G$, we have $|\operatorname{Cent}(G)| \geq|\operatorname{Cent}(M)| \geq p_{1}^{t}+2$ which is equal or greater than $p^{m}+2$ by hypothesis. This proves the claim. Now we want to find the structure of nonnilpotent groups $G$ for which the equality occurs.

Assume that $|\operatorname{Cent}(G)|=p^{m}+2$. We shall prove that $\frac{G}{Z(G)} \cong\left(Z_{p}\right)^{m} \rtimes Z_{l}$ for some positive integer $l$. By hypothesis and the previous paragraph, there is a subgroup $M$ of $G$ such that $\frac{M}{Z(M)}$ is Frobenius with the kernel $\frac{K}{Z(M)}$ of order $p^{m}$ and the Frobenius complement $\frac{H}{Z(M)}$ which is cyclic of prime order. Since $\left|\frac{K}{Z(M)}\right|+|\operatorname{Cent}(K)| \leq|\operatorname{Cent}(M)|$ by Proposition 2.4 and $|\operatorname{Cent}(M)| \leq|\operatorname{Cent}(G)|=p^{m}+2$, we have $|\operatorname{Cent}(M)|=p^{m}+2$ and $|\operatorname{Cent}(K)|=1$. It follows that $K$ is abelian and so $M$ is a $C A$-group by Theorem A (II) of [14]. Next, we show that $G$ is a $C A$-group.

Since $M$ is a $C A$-group, we have $p^{m}+1$ is the maximum size of a set of pairwise non-commuting elements of $M$ by Lemma 2.6 of [1] and so the maximum size of a set of pairwise non-commuting elements of $G$ is at least $p^{m}+1$. Since $|\operatorname{Cent}(G)|=p^{m}+2$, the maximum size of a set of pairwise non-commuting elements of $G$ must be $p^{m}+1$. Therefore $G$ is $C A$-group by Lemma 2.6 of [1]. Now we apply Theorem A of [14].

Note, first, that if 8 divides $|G|=n$, then by hypothesis $|\operatorname{Cent}(G)| \leq \mid \operatorname{Cent}\left(D_{8} \times\right.$ $\left.Z_{\frac{n}{8}}\right) \mid=4$ and so $|\operatorname{Cent}(G)|=4$. Therefore $\frac{G}{Z(G)} \cong Z_{2} \times Z_{2}$ by Fact 3 of [7] and so $G$ is nilpotent, a contradiction. Hence 8 does not divide $n$ and so $|\operatorname{Cent}(G)| \geq 5$ by Fact 4 of [7]. Also if 6 divides $n$, then $|\operatorname{Cent}(G)| \leq\left|\operatorname{Cent}\left(D_{6} \times Z_{\frac{n}{6}}\right)\right|=5$ which implies $|\operatorname{Cent}(G)|=5$. Therefore $\frac{G}{Z(G)} \cong D_{6}$ by Fact [7] and so we have the result. Thus we may assume that 6 does not divide $n$. Now since $G$ is not nilpotent, $G$ satisfies (I), (II) or (III) of Theorem A of [14]. Therefore $G$ has an abelian subgroup $A$ of prime index $r$ or $\frac{G}{Z(G)}=\frac{K}{Z(G)} \rtimes \frac{T}{Z(G)}$ is a Frobenius group with the Frobenius kernel $\frac{K}{Z(G)}$ and the Frobenius complement $\frac{T}{Z(G)}$. In the first case, we have $\left|G^{\prime}\right|=p^{m}$ by Theorem 2.3 of [6] and so $\left|\frac{G}{Z(G)}\right|=p^{m} r$ by Lemma 4 (page 303) of [8]. Consequently $\frac{G}{Z(G)}=\frac{A}{Z(G)} \rtimes \frac{L}{Z(G)}$ where $\left|\frac{L}{Z(G)}\right|=r$ and $\left|\frac{A}{Z(G)}\right|=p^{m}$. By the property of $p^{m}$, the number of Sylow $r$ subgroup of $\frac{G}{Z(G)}$ is $p^{m}$ and so $\frac{G}{Z(G)}$ is Frobenius. Again by the property of $p^{m}, \frac{A}{Z(G)}$ is characteristically simple which implies that it is elementary, as wanted.

In the second case, it follows from (II)-(III) of Theorem A of [14] that $T$ is abelian and $K$ is abelian or $K=Q Z(G)$ where $Q$ is a normal Sylow $q$-subgroup of $G$ for some prime $q$. If $K$ is abelian, then $Z(G)<Z(K)$ and so $|\operatorname{Cent}(G)|=\left|\frac{K}{Z(G)}\right|+2$ by Proposition 2.4. Therefore $\left|\frac{K}{Z(G)}\right|=p^{m}$. By the property of $p^{m}, \frac{K}{Z(G)}$ is elementary. On the other hand $\frac{T}{Z(G)}$ is cyclic by Corollary 6.17 of [15] and so we have the result.

If $K=Q Z(G)$, then $\left|\frac{K}{Z(G)}\right|=q^{a}$ and since $\frac{G}{Z(G)}$ is Frobenius, we have $\left|\frac{T}{Z(G)}\right|$ divides $q^{a}-1$. It follows that $p^{m} \leq q^{a}$ by hypothesis. On the other hand $p^{m}+2=|\operatorname{Cent}(G)| \geq$ 
$\left|\frac{K}{Z(G)}\right|+2$ by Proposition 2.4 and this implies that $p^{m}=q^{a}$. It follows from Proposition 2.4 that $|\operatorname{Cent}(K)|=1$ and $K$ is abelian. The rest of the proof is similar to the previous case.

\section{Proof of Theorem 1.4}

The proof is similar to the previous theorem. If $G$ is nilpotent, then $\omega(G) \geq p+1$ where $p$ is the smallest prime such that $p^{3}$ divides $|G|$ and the equality holds if and only if $\frac{G}{Z(G)} \cong Z_{p} \times Z_{p}$.

Now suppose that $G$ is nonnilpotent. Then $G$ contains a minimal nonnilpotent subgroup $M$ and so $\frac{M}{Z(M)}=\frac{K}{Z(M)} \rtimes \frac{H}{Z(M)}$ is Frobenius such that $\left|\frac{K}{Z(M)}\right|=p_{1}^{m}$ and $\left|\frac{H}{Z(M)}\right|=p_{2}$ by Lemma 2.3. Since $H$ is abelian and has at least $p_{1}^{m}$ conjugates in $G$, say $H=H_{1}, H_{2}, \cdots, H_{p_{1}^{m}}$, we see $\left\{x_{1}, \cdots, x_{p_{1}^{m}}\right\}$ is a subset of pairwise noncommuting elements of $M$ where $x_{i} \in H_{i} \backslash\{1\}$. It follows that $\omega(G) \geq p_{1}^{m}+1 \geq p^{m}+1$. The remainder of the proof is similar to Theorem 1.1.

Acknowledgment. The authors would like to thank the referee for his $/$ her careful reading and valuable comments.

\section{References}

[1] A. Abdollahi, S. M. Jafarian. Amiri and A. M. Hassanabadi, Groups with specific number of centralizers, Houston J. Math. 33(1) (2007), 43-57.

[2] A. Abdollahi, S. Akbari and H. R. Maimani, Non-commuting graph of a group, J. Algbera 298 (2) (2006), 468-492

[3] A. Abdollahi, A. Azad, A. Mohamadi Hasanabadi and M. Zarrin, On the clique numbers of non-commuting graphs of certain groups, Algebra Colloq, 17(4) (2010), 611-620.

[4] A. R. Ashrafi, On finite groups with a given number of centralizers, Algebra Colloq. 7(2) (2000), 139-146.

[5] A. Ballester-Bolinches and J. Cossey, On non-commuting sets in finite soluble CC-groups, Publ. Mat. 56 (2012), 467-471

[6] S. J. Baishya, On finite groups with specific number of centralizers, International Electronic Journal of Algebra, 13(2013), 53-62.

[7] S. M. Belcastro and G. J. Sherman, Counting centralizers in finite groups, Math. Mag. 5 (1994), 111-114.

[8] Y. G. Berkovich and E. M. Zhmu'd, Characters of Finite Groups, Part 1, Transl. Math. Monographs 172, Amer. Math. Soc., Providence. RI, 1998.

[9] R. Brown, Minimal covers of $S_{n}$ by abelian subgroups and maximal subsets of pairwise noncommuting elements, J. Combin. Theory Ser. A 49 (1988), 294-307.

[10] R. Brown, Minimal covers of $S_{n}$ by abelian subgroups and maximal subsets of pairwise noncommuting elements, II, J. Combin. Theory Ser. A 56 (1991), 285-289.

[11] E. A. Bertram, Some applications of graph theory to finite groups, Discrete Math. 44(1) (1983), 31-43

[12] A. M. Y. Chin, On noncommuting sets in an extraspecial p-group, J. Group Theory 8(2) (2005), 189-194.

[13] A. Y. M. Chin, On non-commuting sets in an extraspecial p-group, J. Group Theory, 8.2 (2005), 189-194.

[14] S. Dolfi, M. Herzog and E. Jabara, Finite groups whose noncentral commuting elements have centralizers of equal size, Bull. Aust. Math Soc, 82 (2010), 293-304.

[15] I. M. Isaacs, Finite group theory, Grad. Stud. Math, vol. 92, Amer. Math. Soc, Providence, RI, 2008.

[16] The GAP Group, GAP-Groups, Algoritms, and Programming, version 4.4.10, (2007) ,(http://www.gap-system.org).

[17] S. M. Jafarian Amiri and H. Madadi, On the maximum number of the pairwise noncommuting elements in a finite group, J. Algebra Appl, (2016), Vol. 16, No. 1 (2017) 1650197 (9 pages). 
[18] S. M. Jafarian Amiri, H. Madadi and H. Rostami, On 9-centralizer groups, J. Algebra Appl, Vol. 14, No. 1 (2015) 1550003 (13 pages).

[19] S. M. Jafarian Amiri and H. Rostami, Groups with a few nonabelian centralizers, Publ. Math. Debrecen, 87 (3-4) (2015), 429-437.

[20] S. M. Jafarian Amiri, H. Madadi and H. Rostami, On F-groups with central factor of order $p^{4}$, Math. Slovaca, Accepted.

[21] S. M. Jafarian Amiri, M. Amiri and H. Rostami, Finite groups determined by the number of element centralizers, Comm. Alg., 45(9) (2017), 3792-3797.

[22] B. H. Neumann, A problem of Paul Erdös on groups, J. Austral. Math. Soc. Ser. A 21 (1976), 467-472.

[23] J. Pakianathan, S. Krishnan Shankar, Nilpotent numbers, Amer. Math. Monthly, (2000), 631-634.

[24] L. Pyber, The number of pairwise noncommuting elements and the index of the centre in a finite group, J. Lond. Math. Soc. 35(2) (1987), 287-295.

[25] D. J. S. Robinson, A course in the theory of groups, Springer-Verlag New York, 1996.

[26] R. Schmidt, Zentralisatorverbände endlicher Gruppen, Rend. Sem. Mat. Univ. Padova 44 (1970), 97-131.

[27] M. Zarrin, Criteria for the solubility of finite groups by its centralizers, Arch. Math. 96 (2011), 225-226.

[28] M. Zarrin, On element centralizers in finite groups, Arch. Math. 93(2009), 497-503.

[29] M. Zarrin, On solubility of groups with finitely many centralizers, Bull. Iran. Math. Soc. 39 (2013), 517-521. 